\title{
ALMA OBSERVATIONS OF THE MASSIVE MOLECULAR OUTFLOW G331.512-0.103
}

\author{
Manuel Merello $^{1,2}$, Leonardo Bronfman ${ }^{1}$, Guido Garay ${ }^{1}$, Nadia Lo ${ }^{1}$, Neal J. Evans II $^{2}$, \\ LaRs-ÅKe Nyman ${ }^{3}$, Juan R. Cortés ${ }^{3}$, and Maria R. Cunningham ${ }^{4}$ \\ ${ }^{1}$ Departamento de Astronomía, Universidad de Chile, Casilla 36-D, Santiago, Chile \\ 2 Department of Astronomy, The University of Texas at Austin, 2515 Speedway, Stop C1400, Austin, TX 78712-1205, USA \\ ${ }^{3}$ Joint ALMA Observatory (JAO), Alonso de Cordova 3107, Vitacura, Santiago, Chile \\ ${ }^{4}$ School of Physics, University of New South Wales, Sydney, NSW 2052, Australia \\ Received 2013 June 14; accepted 2013 July 22; published 2013 August 14
}

\begin{abstract}
The object of this study is one of the most energetic and luminous molecular outflows known in the Galaxy, G331.512-0.103. Observations with ALMA Band $7(350 \mathrm{GHz} ; 0.86 \mathrm{~mm})$ reveal a very compact, extremely young bipolar outflow and a more symmetric outflowing shocked shell surrounding a very small region of ionized gas. The velocities of the bipolar outflow are about $70 \mathrm{~km} \mathrm{~s}^{-1}$ on either side of the systemic velocity. The expansion velocity of the shocked shell is $\sim 24 \mathrm{~km} \mathrm{~s}^{-1}$, implying a crossing time of about $2000 \mathrm{yr}$. Along the symmetry axis of the outflow, there is a velocity feature, which could be a molecular "bullet" of high-velocity dense material. The source is one of the youngest examples of massive molecular outflow found associated with a high-mass star.
\end{abstract}

Key words: ISM: jets and outflows - ISM: molecules - stars: formation

Online-only material: color figures

\section{INTRODUCTION}

The formation of massive stars has a major impact on the surrounding interstellar medium, and regions of massive star formation dominate the star formation appearance of galaxies. While during the last couple of decades significant progress (both observational and theoretical) has been made in the study of massive stars and their parental molecular clouds, we are still far away from a complete picture of their formation process (for a recent discussion about this subject, see Kennicutt \& Evans 2012).

Massive stars rapidly affect their surroundings, so catching one early in the formation process can provide valuable insights. Since they form rarely compared with their low-mass counterparts, most are at large distances, making high-resolution observations challenging. The Atacama Large Millimeter/submillimeter Array (ALMA) provides the capability of studying distant objects in the Galaxy with good spatial resolution and sensitivity.

The G331.5-0.1 molecular cloud is a giant, elongated (178 by $41 \mathrm{pc}$ ) cloud in the Norma spiral arm, at a distance of $7.5 \mathrm{kpc}$, with a total $\mathrm{CO}$ mass of $8.7 \times 10^{6} M_{\odot}\left(M_{\mathrm{LTE}}=\right.$ $3.5 \times 10^{6} M_{\odot}$ from $\mathrm{C}^{18} \mathrm{O}$ emission) and a total infrared luminosity of $3.6 \times 10^{6} L_{\odot}$ (Bronfman et al. 2008; Merello et al. 2013), making it one of the most active and extreme high-mass star-forming environments in our Galaxy. The central region hosts an extended $\mathrm{H}_{\mathrm{II}}$ region/infrared source and a number of ultracompact H II (UCHII) regions. Maps at submillimeter wavelengths show six continuum sources, with masses above $10^{3} M_{\odot}$ and average gas surface densities of $0.4 \mathrm{~g} \mathrm{~cm}^{-2}$. Single dish spectral line observations with the Atacama Submillimeter Telescope Experiment and the Atacama Pathfinder EXperiment (APEX) of one of these sources, MM3, revealed extremely broad ( $\pm 80 \mathrm{~km} \mathrm{~s}^{-1}$ ) wings on lines of CO, CS (Bronfman et al. 2008), SO, and SiO (Merello et al. 2013). This highly massive and energetic outflow, G331.512-0.103, is unresolved spatially in a $7^{\prime \prime}$ beam.

Here we present observations with ALMA that resolve the G331.512-0.103 molecular outflow, allowing derivation of the outflow properties including age. In forthcoming papers, we will describe the full set of molecular lines observed with ALMA Band 7.

\section{OBSERVATIONS AND RESULTS}

The observations were performed on 2012 January 12 at frequencies around $350 \mathrm{GHz}(0.86 \mathrm{~mm})$ with ALMA Band 7 in Cycle 0 with 17 (12 m diameter) antennas in the compact configuration, with baselines ranging from $18.5 \mathrm{~m}$ to $269 \mathrm{~m}$. We observed a single field of view centered at $\alpha_{2000}=16^{\mathrm{h}} 12^{\mathrm{m}} 10^{\mathrm{s}} .09$, $\delta_{2000}=-51^{\circ} 28^{\prime} 38^{\prime \prime} .4$. The primary beam was $17^{\prime \prime} .8$ and the synthesized beam was $1.38 \times 0$.' 68 , with a position angle (P.A.) of -37.6 . The integration time was 34 minutes on source, with an additional 24 minutes on calibrators. The nearby quasar J1604-446 was used for flux calibration, and J1427-421, located at 27.7 from the G331.512-0.103 source, was used for phase and bandpass calibrations. The data were processed using the Common Astronomy Software Application (CASA; McMullin et al. 2007), and the resulting maps have cells with sizes of 0.14 , with 254 cells in each spatial dimension. Our images were obtained considering a "briggs" weighting mode on the data with robust $=0.5$. The four spectral windows have a bandwidth of $1875 \mathrm{MHz}$ containing 3840 channels. The separation between each channel is $488.281 \mathrm{kHz}\left(0.4215 \mathrm{~km} \mathrm{~s}^{-1}\right.$ at $357.3 \mathrm{GHz}$ ). The bands were centered at $345.79599 \mathrm{GHz}$, $347.2 \mathrm{GHz}, 357.3 \mathrm{GHz}$, and $358.60587 \mathrm{GHz}$.

While a rich spectrum of lines was observed, this Letter focuses on the lines of $\mathrm{SiO}(8-7)(347.33058 \mathrm{GHz}), \mathrm{H}^{13} \mathrm{CO}^{+}(4-3)$ $(346.99835 \mathrm{GHz}), \mathrm{HCO}^{+}(4-3)(356.73424 \mathrm{GHz})$, and $\mathrm{CO}(3-2)$ $(345.79599 \mathrm{GHz})$, mostly diagnosing the outflow and its interaction with the surrounding cloud. Table 1 presents the four lines reported in the present work, the noise for each, the peak flux density, and the velocity at the peak. The rms noise was assessed over a region of $35^{\prime \prime} \times 35^{\prime \prime}$ centered on the source, avoiding a square area of $11^{\prime \prime}$ in size centered on the emission. For line-free channels, the noise was $\sim 0.014 \mathrm{Jy}_{\text {beam }}{ }^{-1}$. However, for strong emission lines, the noise is larger, presumably because of incomplete $u v$ coverage. On the channels of maximum intensity, 
Table 1

Parameters of the Molecular Line Observations

\begin{tabular}{|c|c|c|c|c|c|c|c|}
\hline Line & $\begin{array}{l}\text { Frequency } \\
\quad(\mathrm{GHz})\end{array}$ & $\begin{array}{c}\text { Noise } \\
\left(\mathrm{Jy} \mathrm{beam}^{-1}\right)\end{array}$ & $\begin{array}{l}\text { Velocity at Peak } \\
\qquad\left(\mathrm{km} \mathrm{s}^{-1}\right)\end{array}$ & $\begin{array}{l}\text { Peak Flux Density } \\
\text { (Jy) }\end{array}$ & $\begin{array}{c}\text { Deconv. Angular Size }{ }^{\mathrm{a}} \\
\left({ }^{\prime \prime}\right)\end{array}$ & $\begin{array}{c}\text { Position Angle } \\
\left({ }^{\circ}\right)\end{array}$ & $\begin{array}{l}\text { Deconv. Size }{ }^{\mathrm{a}} \\
(\mathrm{pc})\end{array}$ \\
\hline $\mathrm{SiO}(8-7)$ & 347.33058 & 0.139 & -89.5311 & 40.4 & $2.6 \times 2.3$ & 105 & 0.09 \\
\hline $\mathrm{H}^{13} \mathrm{CO}^{+}(4-3)$ & 346.99835 & 0.045 & -91.9158 & 30.1 & $3.5 \times 2.4$ & 93 & 0.11 \\
\hline $\mathrm{HCO}^{+}(4-3)$ & 356.73424 & 0.214 & -85.0056 & 91.4 & $2.6 \times 2.5$ & 106 & 0.09 \\
\hline $\mathrm{CO}(3-2)$ & 345.79599 & 0.365 & -96.9012 & 238.5 & $2.8 \times 2.5$ & 110 & 0.10 \\
\hline
\end{tabular}

Note. ${ }^{a}$ FWHM of moment 0 maps, integrated over full velocity extension of the line.

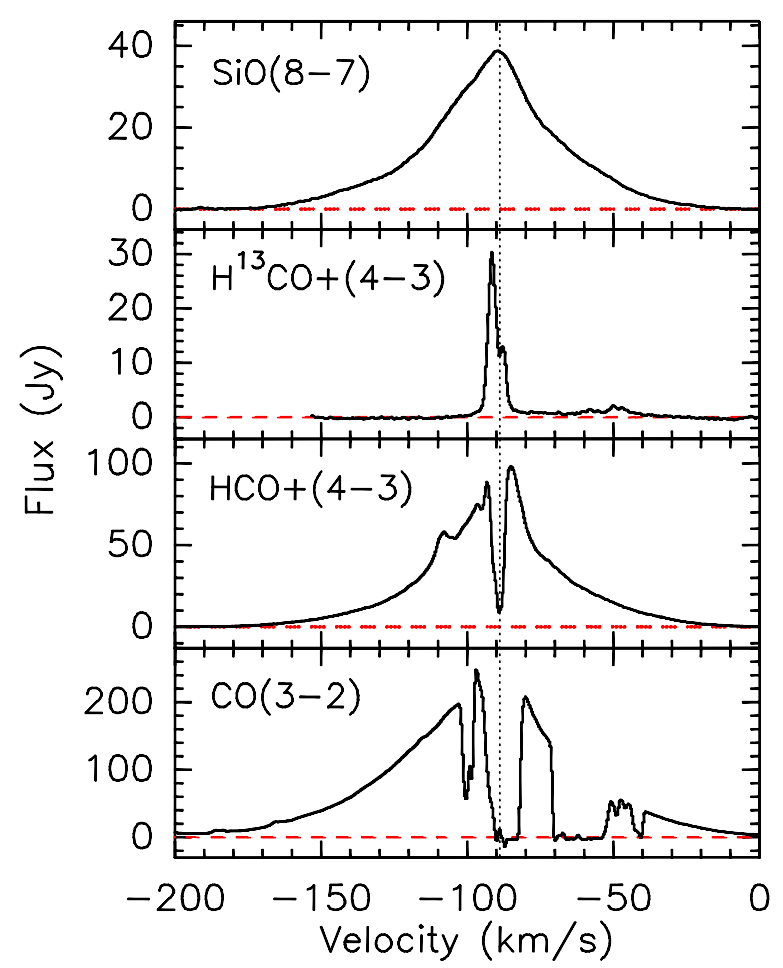

Figure 1. Integrated spectra observed with ALMA of the G331.512-0.103 molecular outflow. A dashed line is drawn in each spectrum representing the baseline. The dotted vertical line shows the systemic velocity of this source $\left(-88.9 \mathrm{~km} \mathrm{~s}^{-1}\right)$.

(A color version of this figure is available in the online journal.)

toward the systemic velocity of the source, the noise increases substantially. To be conservative, we use the noise values at the line velocities in setting thresholds for contours, etc. Moment 0 maps were obtained for each emission line over its full velocity extension, and FWHM angular sizes and P.A.s of these maps were fitted using the task IMFIT of CASA (Columns 6 and 7 of Table 1). Physical sizes, considering a source distance of $7.5 \mathrm{kpc}$, are presented in Column 8 of Table 1.

Figure 1 shows the integrated spectra for the lines described in Table 1. The integration is made over a box of $10^{\prime \prime} .5 \times 10^{\prime \prime} .5$, centered at $\alpha_{2000}=16^{\mathrm{h}} 12^{\mathrm{m}} 09^{\mathrm{s}} .965, \delta_{2000}=-51^{\circ} 28^{\prime} 38^{\prime \prime} .250$. Very wide wings (up to $\pm 70 \mathrm{~km} \mathrm{~s}^{-1}$ ) are seen on the $\mathrm{SiO}$ $J=8 \rightarrow 7$, CO $J=3 \rightarrow 2$, and $\mathrm{HCO}^{+} J=4 \rightarrow 3$ lines. The $\mathrm{H}^{13} \mathrm{CO}^{+} J=4 \rightarrow 3$ line is narrow toward the systemic velocity of the source $\left(-88.9 \mathrm{~km} \mathrm{~s}^{-1}\right)$, but also shows traces of emission over the red wing of the outflow. The high-velocity wings in the $\mathrm{SiO}$ profile match those in the APEX spectra presented in Merello et al. (2013), with differences between both profiles of less than $10 \%$, indicating that the ALMA observations recover all the emission seen in the single-dish spectra. The $\mathrm{CO}$ spectrum presents several absorption features at different velocities. Besides the self-absorption component at the systemic velocity of the source, an absorption dip at $\sim-100 \mathrm{~km} \mathrm{~s}^{-1}$ is related to a second velocity component in the central region of the G331.5-0.1 giant molecular cloud, while the large feature between -70 and $-52 \mathrm{~km} \mathrm{~s}^{-1}$ and the one at $-40 \mathrm{~km} \mathrm{~s}^{-1}$ are related to foreground galactic emission identified in Bronfman et al. (1989). The self-absorption dip at $-88.9 \mathrm{~km} \mathrm{~s}^{-1}$ is also present in the $\mathrm{HCO}^{+}$profile and weakly in the $\mathrm{H}^{13} \mathrm{CO}^{+}$profile.

The $\mathrm{H}^{13} \mathrm{CO}^{+}$profile resembles a typical blue profile found in regions with infalling material (Myers et al. 2000), but observations of a more optically thin molecule, like $\mathrm{HC}^{18} \mathrm{O}^{+}$, will be needed to test this hypothesis. The $\mathrm{H}^{13} \mathrm{CO}^{+}$profile also shows a trace of emission in the red wing of the outflow, with a secondary peak of emission at $\sim-50 \mathrm{~km} \mathrm{~s}^{-1}$.

Figure 2 shows the channel maps of the $\mathrm{SiO}$ line emission. The crosses indicate the peak positions at velocities about $\pm 40 \mathrm{~km} \mathrm{~s}^{-1}$ from the systemic velocity. These channels define a "bipolar axis" of symmetry of $102.5 \pm 1$.6. Experiments using other channels, different signal-to-noise levels, etc. resulted in variations around this value of only 1.6 , which we assign as the uncertainty. This angle differs slightly from the P.A. found in the deconvolved moment 0 map of $\mathrm{SiO}$, (P.A. of $105^{\circ}$; see Table 1).

The integrated intensity $\mathrm{SiO}$ map shows a ring-type feature toward the systemic velocity of the source $\left(-90 \pm 15 \mathrm{~km} \mathrm{~s}^{-1}\right)$. We used the $3 \sigma$ contour level to estimate the outer edge of this emission, obtaining major and minor axes of 5". $12 \pm 0$ ". 16 and $4^{\prime \prime} .52 \pm 0.33$, respectively, with a P.A. of 140.6 . The deconvolved sizes are then $4^{\prime \prime} .79 \pm 0^{\prime \prime} .19$ and $4^{\prime \prime} .42 \pm 0$.'35 (0.17 pc at the source distance), with a P.A. of $137^{\circ}$.

The P.A. of the beam elongation is $175^{\circ}$ from this angle, so some of the extension may be beam-related, but the extent is considerably larger than the beam, and the deconvolution should remove that effect. The inner cavity is centered at $\alpha_{2000}=16^{\mathrm{h}} 12^{\mathrm{m}} 10^{\mathrm{s}} .00, \delta_{2000}=-51^{\circ} 28^{\prime} 37^{\prime \prime} .45$, it has a size of $0{ }^{\prime} 99 \times 00^{\prime \prime} 66$, with a P.A. of $160^{\circ}$, and it is determined by the $16 \sigma$ emission contour. Therefore, the cavity in the $\mathrm{SiO}$ emission at the systemic velocity is not resolved with the present beam size. The $\mathrm{SiO}$ map also shows clumpy, irregular structure at the systemic velocity, with two peaks of emission of 5.0 and 3.9 $\mathrm{Jy} \mathrm{beam}^{-1}$, located symmetrically at both sides of the inner cavity.

Figure 3 shows the intensity map of $\mathrm{H}^{13} \mathrm{CO}^{+}$in color, overlaid with $\mathrm{SiO}$ contours in black. The line maps are at $\sim-91.9 \mathrm{~km} \mathrm{~s}^{-1}$, near the systemic velocity of the source, where the $\mathrm{H}^{13} \mathrm{CO}^{+}$ profile shows the peak of intensity. The $\mathrm{H}^{13} \mathrm{CO}^{+}$emission shows a ring shape similar to that seen in $\mathrm{SiO}$, with a inner hole coincident with the cavity found in the $\mathrm{SiO}$ observations, but with most of this emission lying outside the $\mathrm{SiO}$ ring. The $\mathrm{H}^{13} \mathrm{CO}^{+}$inner hole has a size of $1^{\prime \prime} .82 \times 1^{\prime \prime}$. 12 , with a P.A. of 


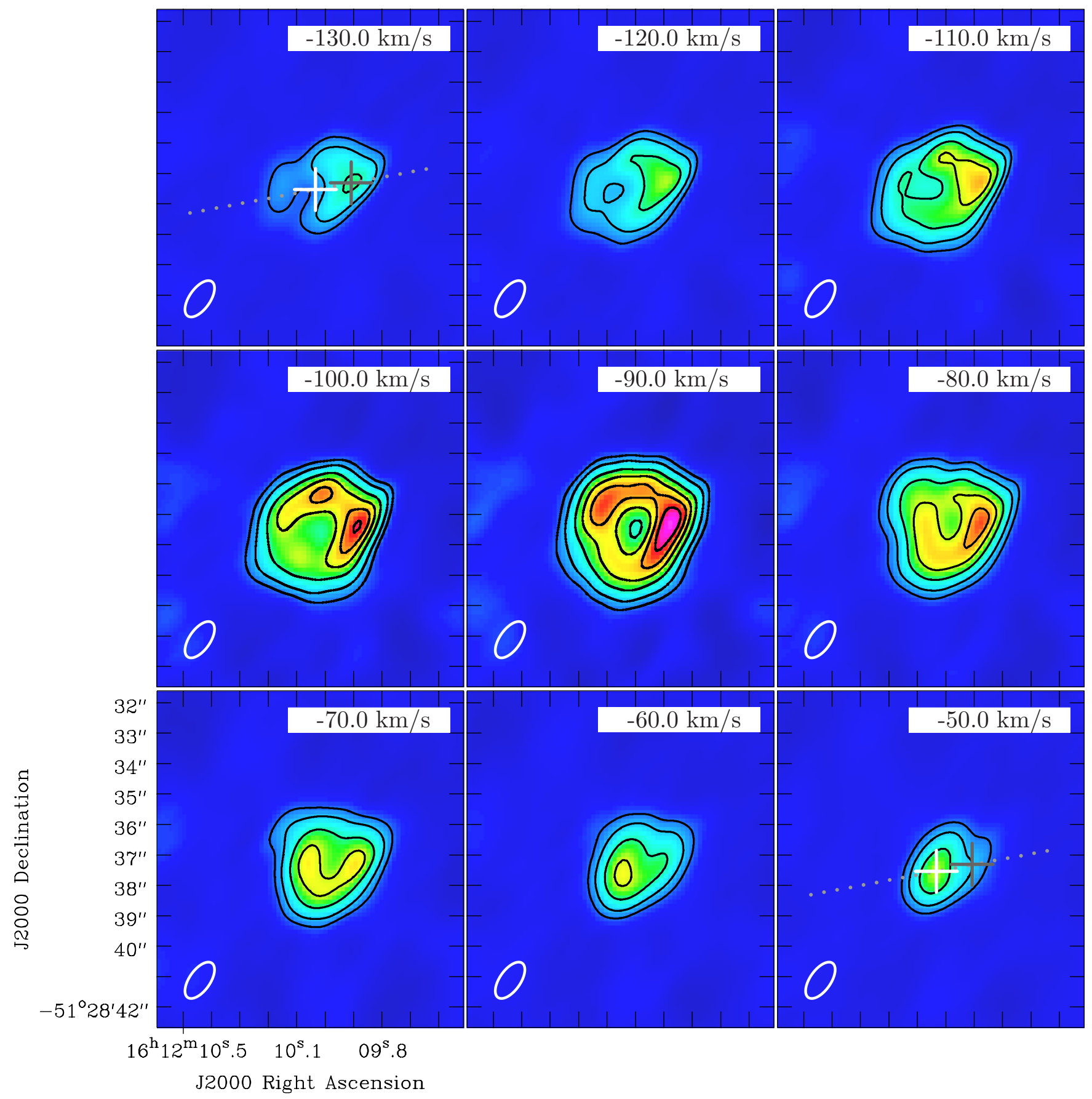

Figure 2. Channel maps of the $\mathrm{SiO}(8-7)$ line emission. The contours are at 3, 6, 12, 18, 24, and 30 times the rms defined for the $\mathrm{SiO}$ emission $\left(0.14 \mathrm{Jy}\right.$ beam $\left.{ }^{-1}\right)$. The velocity of each channel is indicated in the upper right corner of each box. The channels are separated by $10 \mathrm{~km} \mathrm{~s}^{-1}$. In the central channel map, toward the systemic velocity of the source $\left(-88.9 \mathrm{~km} \mathrm{~s}^{-1}\right)$, the emission shows a clear ring-type or projected shell structure, with the inner hole well determined at the $18 \sigma$ contour. The ALMA synthesized beam is shown at the bottom left corner in each box. The gray and white crosses define the positions considered in the blue and red lobes to set the symmetry axis of the outflow, represented by the gray dotted line.

$112^{\circ}$, and it is determined by the $4 \sigma$ emission contour. The $\mathrm{H}^{13} \mathrm{CO}^{+}$structure observed at this velocity channel has major and minor axes, considering the $3 \sigma$ contour as the outer edge of this emission, of $8^{\prime \prime} .10 \pm 0^{\prime \prime} .32$ and $6.79 \pm 0.20$, respectively, with a P.A. of $78^{\circ}$. The deconvolved angular sizes are then $8^{\prime \prime} .03 \pm 0.32$ and $6.57 \pm 0.22$, with a P.A. of $76^{\circ}$, which correspond to a geometric mean size of $0.26 \mathrm{pc}$ at the source distance.

The red ellipse in Figure 3 represents the 50\% contour of the radio continuum source at $8.6 \mathrm{GHz}$ (Merello et al. 2013). The emission at $8.6 \mathrm{GHz}$ is located inside the inner cavity of the $\mathrm{SiO}$ emission and is unresolved within the beam $\left(1^{\prime \prime} .51 \times 0.99\right)$.

\section{DISCUSSION}

The $\mathrm{SiO}$ emission is an indicator of shocked gas, commonly seen around young stellar objects, with an enhanced abundance attributed to Si being sputtered from dust grains for jet velocities exceeding $25 \mathrm{~km} \mathrm{~s}^{-1}$ (e.g., Arce et al. 2007; Garay et al. 1998, 2002). Because the wing velocities of the G331.512-0.103 outflow extend to $\pm 70 \mathrm{~km} \mathrm{~s}^{-1}$, we expected to see $\mathrm{SiO}$ emission in the wings, likely with a bipolar pattern, and we do. However, the fact that a ring of $\mathrm{SiO}$ emission is also present at the systemic velocity, with a cavity of the size of the beam, indicates that 

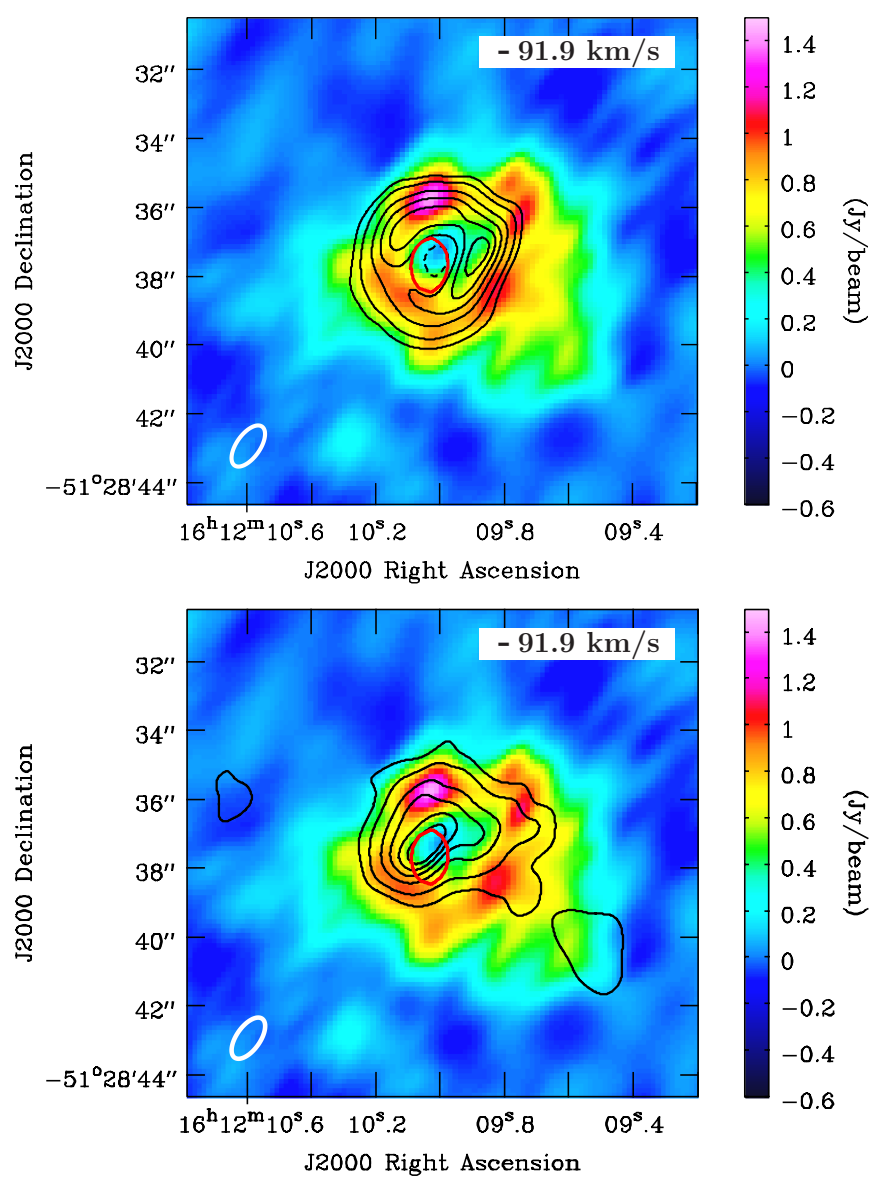

Figure 3. Top: $\mathrm{H}^{13} \mathrm{CO}^{+}(4-3)$ emission (in colors) of the G331.512-0.103 source at a velocity of $-91.9 \mathrm{~km} \mathrm{~s}^{-1}$, where the peak of flux density is found. The overlaid contours in black correspond to the $\mathrm{SiO}(8-7)$ emission at the same velocity, at $3 \sigma, 6 \sigma, 12 \sigma, 18 \sigma, 24 \sigma$, and $30 \sigma$ (with $\sigma=0.14 \mathrm{Jy} \mathrm{beam}^{-1}$ ). The dashed contour represents the $12 \sigma$ emission toward the cavity observed in $\mathrm{SiO}$, which is coincident spatially with the ones observed with $\mathrm{H}^{13} \mathrm{CO}^{+}$. The red contour shows the $50 \%$ of emission (peak of $158 \mathrm{mJy}^{\text {beam }}{ }^{-1}$ ) of the radio source detected at $8.6 \mathrm{GHz}$ toward this source, presented by Merello et al. (2013). The peak position of the radio continuum observations is inside the cavity described by the $\mathrm{SiO}$ and $\mathrm{H}^{13} \mathrm{CO}^{+}$emissions. Bottom: $\mathrm{H}^{13} \mathrm{CO}^{+}(4-3)$ emission (in colors), with overlaid black contours of the $0.86 \mathrm{~mm}$ continuum emission obtained with ALMA. The contours are at $3 \sigma, 6 \sigma, 12 \sigma, 18 \sigma, 24 \sigma$, $30 \sigma$ (with $\sigma=0.02 \mathrm{Jy} \mathrm{beam}^{-1}$ ). The continuum emission peaks, within errors, in the center of the cavity shown in the $\mathrm{H}^{13} \mathrm{CO}^{+}$emission.

a more isotropic high-speed wind is producing shocked gas in a shell. We interpret then that the G331.512-0.103 source corresponds to a jet almost in the line of sight with an expanding shocked shell surrounding its driving source, which in projection is observed as a ring at the systemic velocity.

To explore this hypothesis, we construct position-velocity (PV) diagrams of the $\mathrm{SiO}$ and $\mathrm{H}^{13} \mathrm{CO}^{+}$emission (Figure 4), for slices along the bipolar axis and perpendicular to it. The cavity is clearly present in all diagrams, centered near $v=-90 \mathrm{~km} \mathrm{~s}^{-1}$. The SiO PV diagram along the secondary axis shows two strong peaks nearly symmetric around the cavity. These two peaks extend around the cavity down to the $16 \sigma$ emission contour (represented in red in Figure 4), extending in velocity between -111.9 and $-63.8 \mathrm{~km} \mathrm{~s}^{-1}$. The PV diagram along the bipolar axis shows a strong peak at an angular offset of $1^{\prime \prime}$. The $3 \sigma$ contour of the $\mathrm{H}^{13} \mathrm{CO}^{+}$traces the red wing of the outflow up to a velocity of $\sim-25 \mathrm{~km} \mathrm{~s}^{-1}$. An additional peak is observed at $-45 \mathrm{~km} \mathrm{~s}^{-1}$ along the primary axis, but at $-50 \mathrm{~km} \mathrm{~s}^{-1}$ along the secondary axis. This emission extends between $-59<v_{\text {lsr }}<-29 \mathrm{~km} \mathrm{~s}^{-1}$, defined by the $6 \sigma$ contour in the $\mathrm{H}^{13} \mathrm{CO}^{+} \mathrm{PV}$ diagram along the symmetry axis, and it has a size of $2^{\prime \prime} .5 \times 1^{\prime \prime} .4(\sim 0.07 \mathrm{pc})$. We consider this high-velocity emission likely to be a molecular "bullet," a feature sometimes observed in star formation regions (e.g., Masson \& Chernin 1993; Santiago-García et al. 2009; Kristensen et al. 2012).

The bipolar symmetry at the extreme velocities requires a jet-like outflow, but the ring structure at the systemic velocity indicates a nearly isotropic component. The latter surrounds a region of ionized material, whose expansion could produce the isotropic component. Considering the extension of the symmetric peaks in the $\mathrm{PV}$ diagram of $\mathrm{SiO}$, we estimate that the expanding shell has a velocity of $\sim 24 \mathrm{~km} \mathrm{~s}^{-1}$. A jet originating from the high-mass forming star, the likely source of the radio emission observed at $8.6 \mathrm{GHz}$, could drive the molecular outflow observed in the high-velocity wings of the $\mathrm{SiO}$ spectra. The $\mathrm{H}^{13} \mathrm{CO}^{+}$emission, being optically thin toward the wings of the outflow, is only tracing the red lobe of this outflow, and the line profile suggests that toward the systemic velocity, some gas is still infalling toward the central star. However, such a dip can also be created by resolving out ambient cloud emission, so further observations would be needed to test this interpretation.

An estimate for the crossing time is given by $t_{\text {cross }}=$ $\theta /\left(2 v_{\max }\right)$, with $\theta$ the diameter of a projected shell, and $v_{\max }$ the maximum velocity relative to the ambient gas. Using $v_{\text {max }}=24 \mathrm{~km} \mathrm{~s}^{-1}$, we obtain $t_{\text {cross }}<600 \mathrm{yr}$ for the inner hole and $\sim 2000 \mathrm{yr}$ for the $\mathrm{SiO}$ ring with diameter $3^{\prime \prime}$ (or $0.11 \mathrm{pc}$, considered as the middle point between the inner and outer edges of the ring). These estimates would make this source one of the youngest examples of flows around massive forming stars. The momentum $\left(2.4 \times 10^{3} M_{\odot} \mathrm{km} \mathrm{s}^{-1}\right)$ and kinetic energy $\left(1.4 \times 10^{48} \mathrm{erg}\right)$ of the outflow were measured by Bronfman et al. (2008) from $\operatorname{CO}(7-6)$ observations. Taking $2000 \mathrm{yr}$ as the relevant time, the resulting force is $1.2 M_{\odot} \mathrm{km} \mathrm{s}^{-1} \mathrm{yr}^{-1}$ and the mechanical luminosity is $6.4 \times 10^{3} L_{\odot}$, both higher than any values found in the compilation of outflow properties by $\mathrm{Wu}$ et al. (2004).

The mass of the $\mathrm{H}^{13} \mathrm{CO}^{+}$emission toward the ambient gas and the bullet is estimated considering a local thermodynamic equilibrium (LTE) formalism and optically thin emission (see, e.g., Garden et al. 1991). Considering an excitation temperature of $100 \mathrm{~K}$, a mean molecular weight $\mu=2.72 m_{\mathrm{H}}$, and an abundance $\left[\mathrm{H}^{13} \mathrm{CO}^{+} / \mathrm{H}_{2}\right]=3.3 \times 10^{-11}$ (Blake et al. 1987), the LTE mass of the ambient gas and the bullet are $36 M_{\odot}$ and $1.8 M_{\odot}$, respectively.

The continuum emission was determined from regions of the ALMA Band 7 spectrum that appeared to be line free, though we cannot rule out some level of contamination by line emission. The lower panel of Figure 3 shows the $0.86 \mathrm{~mm}$ continuum emission, overlaid on the $\mathrm{H}^{13} \mathrm{CO}^{+}$emission at $-91.9 \mathrm{~km} \mathrm{~s}^{-1}$. A point-like source lies within the ring of $\mathrm{SiO}$ and $\mathrm{H}^{13} \mathrm{CO}^{+}$ emission, and a more extended component agrees well with the $\mathrm{H}^{13} \mathrm{CO}^{+}$ring.

The continuum emission has a FWHM of $2^{\prime \prime} .94 \times 2$ ". 18 and a total flux of $4.3 \mathrm{Jy}$. This emission could arise from either free-free emission from a very dense ionized wind or from dust emission, or a combination. As a test, we estimated the integrated flux of the continuum emission with a point source removed at the peak position of the $8.6 \mathrm{GHz}$ continuum emission showed in Figure 3, obtaining a value of $3.9 \mathrm{Jy}$. This integrated flux of the continuum emission is much less than the $66.7 \mathrm{Jy}$ measured at this wavelength in a $33^{\prime \prime}$ region (Merello et al. 2013), but much more than would be expected from a uniformly 

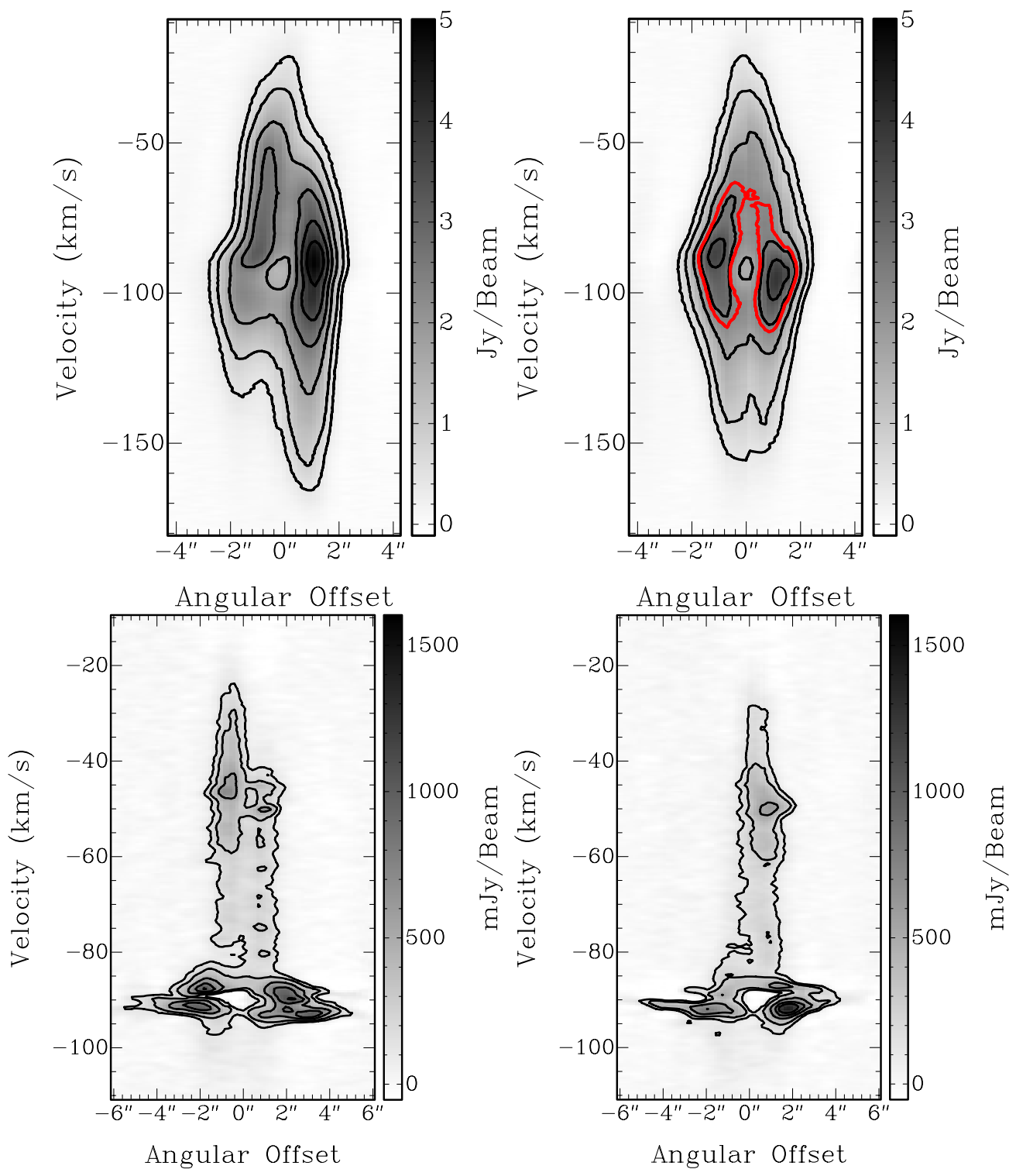

Figure 4. Position-velocity diagrams of the $\mathrm{SiO}(8-7)$ (top) and $\mathrm{H}^{13} \mathrm{CO}^{+}(4-3)$ (bottom) emission of the $\mathrm{G} 331.512-0.103$ source. The slices are centered at the cavity observed in the $\mathrm{SiO}$ map $\left(\alpha_{2000}=16^{\mathrm{h}} 12^{\mathrm{m}} 09^{\mathrm{s}} .985, \delta_{2000}=-51^{\circ} 28^{\prime} 37^{\prime \prime} .42\right)$. Slices along the axis of symmetry of the outflow (primary axis), and perpendicular to this

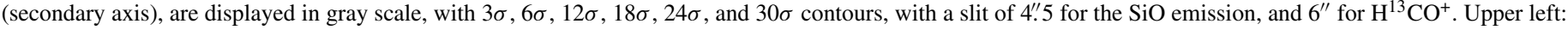
slice of the $\mathrm{SiO}$ emission, running along the axis of symmetry (P.A. of 102.5). Upper right: slice of the $\mathrm{SiO}$ emission, running perpendicular to the axis of symmetry (P.A. of 12.5). An additional $16 \sigma$ contour in red is shown, extending between -111.9 and $-63.8 \mathrm{~km} \mathrm{~s}^{-1}$. Bottom left: slice of the $\mathrm{H}^{13} \mathrm{CO}^{+}$emission, running along the axis of symmetry. Bottom right: slice of the $\mathrm{H}^{13} \mathrm{CO}^{+}$emission, running perpendicular to the axis of symmetry.

(A color version of this figure is available in the online journal.)

distributed emission, therefore we associate this emission with an envelope around the central object. If we attribute this measured flux to dust emission, we can estimate mass and other properties of the source. At a radius of $1^{\prime \prime}$, or $0.036 \mathrm{pc}$ at the source distance, and a luminosity of $7 \times 10^{5} L_{\odot}$ (Merello et al. 2013), the equilibrium dust temperature would be 400-1000 K, for a range of dust properties. Assuming $400 \mathrm{~K}$, and using OH5 opacities, the mass estimated in a region of 2".9 in size, or $0.11 \mathrm{pc}$, would be $38 M_{\odot}$, implying a mass surface density of $5140 M_{\odot} \mathrm{pc}^{-2}$, or $1.07 \mathrm{~g} \mathrm{~cm}^{-2}$, and a mean volume density of $1.3 \times 10^{6} \mathrm{~cm}^{-3}$.

An interesting comparison can be made to the outflow from G5.89 (e.g., Harvey \& Forveille 1988; Acord et al. 1997; Watson et al. 2007; Su et al. 2012), which also shows a shell morphology along with a bipolar flow. The expansion age for the UCHII region is $600 \mathrm{yr}$, measured from multi-epoch observations
(Acord et al. 1998), while the bipolar outflow is older, with estimates ranging from 3000 to $7700 \mathrm{yr}$. Estimates of the outflow properties vary, but the values in the Wu et al. (2004) compilation are a mass of $70 M_{\odot}$, a momentum of $1600 M_{\odot} \mathrm{km} \mathrm{s}^{-1}$, and a mechanical luminosity of $1600 L_{\odot}$. In comparison with the G5.89 source, the G331.512-0.103 outflow has a shorter age and a higher mechanical luminosity and momentum.

\section{SUMMARY AND CONCLUSIONS}

We have performed a study with ALMA Band 7 of the massive and energetic molecular outflow G331.512-0.103. The high angular resolution observations allow us to resolve this source in a set of four molecular lines: $\mathrm{SiO}(8-7), \mathrm{H}^{13} \mathrm{CO}^{+}(4-3)$, $\mathrm{HCO}^{+}(4-3)$, and $\mathrm{CO}(3-2)$. The $\mathrm{SiO}$ emission is confined in a region of a size less than $5^{\prime \prime}(0.18 \mathrm{pc}$ at a distance of $7.5 \mathrm{kpc})$, 
and reveal the presence of a ring-type structure toward the systemic velocity of the source. This feature is also observed in the $\mathrm{H}^{13} \mathrm{CO}^{+}$line, and the cavity is coincident with a strong and compact radio continuum source observed at $8.6 \mathrm{GHz}$. We interpret these observations as a young stellar object producing a compact $\mathrm{H}$ II region, with an expansion shock propagating into the medium and possibly with dense material still infalling around this shell. $\mathrm{CO}, \mathrm{HCO}^{+}$, and $\mathrm{SiO}$ observations trace the full extension of the outflow wings, but the $\mathrm{H}^{13} \mathrm{CO}^{+}$only traces the red wing, also showing the possible presence of a bullet of dense material. We estimated an age of $\sim 2000 \mathrm{yr}$ for the expanding shell, making it one of the youngest examples of massive molecular outflows observed toward massive young stars.

M.M. and N.J.E. gratefully acknowledge support from the NSF grant AST-1109116. L.B. and G.G. acknowledge support from CONICYT project BASAL PFB-06. N.L.'s postdoctoral fellowship is supported by CONICYT/FONDECYT postdoctorado, under project No. 3130540. N.L. acknowledges support from the ALMA-CONICYT Fund for the Development of Chilean Astronomy Project 31090013. This Letter makes use of the following ALMA data: ADS/JAO.ALMA\#2011.0.00524.S. ALMA is a partnership of ESO (representing its member states), NSF (USA), and NINS (Japan), together with NRC (Canada) and NSC and ASIAA (Taiwan), in cooperation with the Republic of Chile. The Joint ALMA Observatory is operated by ESO, AUI/NRAO, and NAOJ.

\section{REFERENCES}

Acord, J. M., Churchwell, E., \& Wood, D. O. S. 1998, ApJL, 495, L107 Acord, J. M., Walmsley, C. M., \& Churchwell, E. 1997, ApJ, 475, 693

Arce, H. G., Shepherd, D., Gueth, F., et al. 2007, in Protostars and Planets V, ed. B. Reipurth, D. Jewitt, \& K. Keil (Tucson, AZ: Univ. Arizona Press), 245

Blake, G. A., Sutton, E. C., Masson, C. R., \& Phillips, T. G. 1987, ApJ, 315,621

Bronfman, L., Alvarez, H., Cohen, R. S., \& Thaddeus, P. 1989, ApJS, 71, 481

Bronfman, L., Garay, G., Merello, M., et al. 2008, ApJ, 672, 391

Garay, G., Köhnenkamp, I., Bourke, T. L., Rodríguez, L. F., \& Lehtinen, K. K. 1998, ApJ, 509, 768

Garay, G., Mardones, D., Rodríguez, L. F., Caselli, P., \& Bourke, T. L. 2002, ApJ, 567,980

Garden, R. P., Hayashi, M., Hasegawa, T., Gatley, I., \& Kaifu, N. 1991, ApJ, 374,540

Harvey, P. M., \& Forveille, T. 1988, A\&A, 197, L19

Kennicutt, R. C., \& Evans, N. J. 2012, ARA\&A, 50, 531

Kristensen, L. E., van Dishoeck, E. F., Bergin, E. A., et al. 2012, A\&A, 542, A8

Masson, C. R., \& Chernin, L. M. 1993, ApJ, 414, 230

McMullin, J. P., Waters, B., Schiebel, D., Young, W., \& Golap, K. 2007, in ASP Conf. Ser. 376, Astronomical Data Analysis Software and Systems XVI, ed. R. A. Shaw, F. Hill, \& D. J. Bell (San Francisco, CA: ASP), 127

Merello, M., Bronfman, L., Garay, G., et al. 2013, ApJ, in press (arXiv:1306.0042)

Myers, P. C., Evans, N. J., II, \& Ohashi, N. 2000, in Protostars and Planets IV, ed. V. Mannings, A. P. Boss, \& S. S. Russell (Tucson, AZ: Univ. Arizona Press), 217

Santiago-García, J., Tafalla, M., Johnstone, D., \& Bachiller, R. 2009, A\&A, 495,169

Su, Y.-N., Liu, S.-Y., Chen, H.-R., \& Tang, Y.-W. 2012, ApJL, 744, L26

Watson, C., Churchwell, E., Zweibel, E. G., \& Crutcher, R. M. 2007, ApJ, 657,318

Wu, Y., Wei, Y., Zhao, M., et al. 2004, A\&A, 426, 503 